\title{
Endemic SARS-CoV-2 will maintain post-pandemic immunity
}

Marc Veldhoen $\mathbb{D}^{1} \bowtie$ and J. Pedro Simas ${ }^{1,2} \bowtie$

COVID-19 vaccinations have started. They will stop the pandemic. Citing recent data that are in line with immunological knowledge and predictions, combined with insights of common cold coronaviruses, we here set out the case that the maintenance of population immunity will not depend on continued vaccinations but on the endemic presence of SARS-CoV-2.

With highly effective vaccines for COVID-19 approved, a critical question for informing health policy in a post-pandemic world is the maintenance of immunity against SARS-CoV-2. One year into the pandemic, a picture has emerged that immune responses generated against SARS-CoV-2 are, on average, in line with immunological predictions for this type of infection. Recent studies show that antibodies to SARS-CoV-2, including neutralizing ones, persist in the serum for at least 6-7 months following infection in $90 \%$ of subjects tested ${ }^{1-5}$. Antibody kinetics are typical of those seen in many other infections. Levels peak 3 weeks post-symptoms with a subsequent contraction and plateau phase. Predicted from the presence of good quality antibodies, $\mathrm{T}$ cell responses are mounted as expected. $\mathrm{T}$ cells are crucial for orchestrating other components of the immune response, including B cell antibody maturation and production and the formation of long-lived memory cells $s^{6,7}$.

What are the implications of these findings for a post-COVID-19 world? Will we achieve protective population-level (or 'herd') immunity? Will vaccinations eradicate SARS-CoV-2? Will repeat vaccinations be required to maintain immunity? We can look to our accumulated knowledge on the ecology of other human coronavirus $(\mathrm{HCoV})$ infections to provide insights into these fundamental questions.

\section{Immunity to human coronaviruses}

There are four known HCoVs that cause asymptomatic or mild upper respiratory tract infections similar to those generated by a collection of upper respiratory tract-targeting virus families. The broad pool of these common cold viruses can make us 'catch a cold' several times a year. The seroprevalence of the common cold $\mathrm{HCoVs}$, initiated in early childhood, is widespread in adults, with reinfections separated by months, mostly without symptoms but with possible viral shedding. Similarly, SARS-CoV-2 infections are overwhelmingly asymptomatic or mild. Mild infections of the upper respiratory tract associate with modest antibody increases and several infection rounds may be required to prevent symptomatic disease. In contrast to endemic CoVs, in the absence of individual and group immunity, SARS-CoV-2 can disseminate to the lungs, and even systemically in some, resulting in pathology and systemic immune responses. $\mathrm{HCoV}$ seroprevalence data and challenge experiments suggest that dissemination to the lower respiratory tract results in more sustained immunity $^{8}$. These observations are in line with animal models, where local and mild infections result in more rapid waning of antibody levels while a severe and systemic CoV strain infection results in long-term immunity. Similarly, disseminating SARS-CoV and MERS-CoV infections evoked systemic responses, with antibodies lasting for a substantial time, 2-5 years ${ }^{8}$. Immune memory cells persist for longer and are able to respond rapidly upon reinfection.

\section{Will vaccines stop the pandemic?}

Although a recently emerged virus, the development of SARS-CoV-2 vaccines is based on decades of knowledge, and we predict that these vaccines will be successful to end the COVID-19 pandemic. Relying on natural infection alone will expose the vulnerable to unacceptable risks and result in differing levels of pathology in a proportion of the population. Effective individual and group protection levels will reduce pathology and disease upon SARS-CoV-2 reinfection. A second important question is whether the vaccines will be effective against reinfection or even eradicate SARS-CoV-2. Here, we suggest both answers are most probably no. Coronavirus vaccines have been used extensively to control infections in domestic animals. Inactivated or intramuscular parenteral vaccines induce high systemic levels of neutralizing antibodies and confer effective protection against disease. However, they have lower efficacy against mucosal $\mathrm{CoV}$ infections and do not prevent viral shedding.

Therefore, SARS-CoV-2 is likely to remain present in the population. The four common cold HCoVs are also thought to have had a more lethal history, possibly incorrectly identified as the cause of influenza pandemics in the past. Continued presence of SARS-CoV-2 is 
akin to endemic HCoVs, where $60-70 \%$ population seroprevalence reflects a spectrum of immunity, dynamically maintained by intermittent reinfection and affords group protection from severe infection in the vulnerable. A large proportion of the population will be protected sufficiently and will reduce the viral shedding burden at any time to prevent large outbreaks. The exception to this will be the reintroduction of the virus into communities in which SARS-CoV-2 is not endemic and/or who are not vaccinated. Infections from a young age and re-infections in later life will build up and maintain immunity. Importantly, those that have not been able to gain immunity via natural infection or vaccination will benefit from herd immunity, despite the virus residing in a largely asymptomatic population.

An important point to reflect on is that the global vaccination programme against SARS-CoV-2 will generate pressure for antigenic escape variants. This has been observed for mucosal CoVs, where antigenic drifts located in spike protein diminish vaccine effectiveness ${ }^{9}$. Intermediate host adaptations may further contribute to the emergence of such variants. This is not a worrying development. A general principle of antigen recognition is that our immune system recognizes multiple different segments of a pathogen. This provides non-overlapping immune protection, already reported for SARS-CoV-2. In contrast to influenza viruses where there is extensive potential of new combinations, only limited changes in the spike protein of CoVs result in the generation of serotype variants. The likely geographical emergence of SARS-CoV-2 antigenic escape variants needs consideration in vaccine design and utilization ${ }^{10}$, but it should not significantly influence achieving protective population immunity. Therefore, with sufficient cross-reactive immunity boosted by regular natural infections that particularly increase mucosal immunity, we suggest it is unlikely that annual SARS-CoV-2 vaccination programmes will be required after the initial years.

\section{The post-pandemic outlook}

We can and should put trust in obtained immunological and viral knowledge and not hesitate to use this to predict a likely future, advise public health measures and support decision-makers. Exact predictions cannot be made but evidence thus far indicates that SARS-CoV-2 behaves very similarly to the existing HCoVs. Anti-SARS-CoV-2 immune responses are in line with expectations of new, potentially disseminating, respiratory tract viruses. Upon disease, immune responses are robust, include neutralizing antibodies and immunological memory, and last for considerable time. Mild or asymptomatic infections likely result in more rapid waning of immunity. Vaccinations will protect from disease and a large proportion of the population will be protected from COVID-19, but this may not prevent re-infection and viral shedding of the respiratory tract $\mathrm{HCoV}$.

A critically important point is that, protection against COVID-19 for the vulnerable is at the population level, not at the level of the individual. Population immunity reduces both the viral spread and burden. This curtails dissemination from the upper respiratory tract, preventing disease, and in turn reduces the infection burden and spread in the population, thereby protecting the vulnerable. Nevertheless, it may not stop viral spread altogether. Hence, SARS-CoV-2 is likely to become the fifth endemic common cold virus, causing largely asymptomatic infections.

Endemic SARS-CoV-2 will ensure maintenance of seroprevalence and mucosal immunity in the population, which will increase over time in new generations. As such, most infected individuals will ultimately endure a largely asymptomatic or mild course of disease, although similarly to the other common cold HCoVs, SARS-CoV-2 may cause fatalities in extremely vulnerable elderly or immunocompromised individuals. SARS-CoV-2 mutants will arise as already reported, but new variants will unlikely differ sufficiently to escape established immunity. Cross-reactive immunity, critically boosted by natural reinfections, should conserve good levels of population protection also against new variants, thereby preventing the occurrence of severe disease, including in the vulnerable. Therefore, we predict that the need for large-scale vaccination programmes will be transient until an endemic state for SARS-CoV-2 is reached.

1. Figueiredo-Campos, P. et al. Seroprevalence of anti-SARS-CoV-2 antibodies in COVID-19 patients and healthy volunteers up to 6 months post disease onset. Eur. J. Immunol. 50, 2025-2040 (2020).

2. Iyer, A. S. et al. Persistence and decay of human antibody responses to the receptor binding domain of SARS-CoV-2 spike protein in COVID-19 patients. Sci. Immunol. 5, eabe0367 (2020).

3. Wajnberg, A. et al. Robust neutralizing antibodies to SARS-CoV-2 infection persist for months. Science 370, 1227-1230 (2020).

4. Ripperger, T. J. et al. Orthogonal SARS-CoV-2 serological assays enable surveillance of low prevalence communities and reveal durable humoral immunity. Immunity 53, 925-933 (2020).

5. Stadlbauer, D. et al. Repeated cross-sectional sero-monitoring of SARS-CoV-2 in New York City. Nature https://doi org/10.1038/ 541586-020-2912-6 (2020)

6. Grifoni, A. et al. Targets of T cell responses to SARS-CoV-2 coronavirus in humans with COVID-19 disease and unexposed individuals. Cell 181, 1489-1501 (2020)

7. Le Bert, N. et al. SARS-CoV-2-specific T cell immunity in cases of COVID-19 and SARS, and uninfected controls. Nature 584 457-462 (2020).

8. Huang, A. T. et al. A systematic review of antibody mediated immunity to coronaviruses: kinetics, correlates of protection, and association with severity. Nat. Commun. 11, 4704 (2020).

9. Lin, S.-Y. \& Chen, H.-W. Infectious bronchitis virus variants: molecular analysis and pathogenicity investigation. Int. J. Mol. Sci. 18, 2030 (2017).

10. Thomson, E. C. et al. The circulating SARS-CoV-2 spike variant N439K maintains fitness while evading antibody-mediated immunity. Preprint at bioRxiv https://doi.org/10.1101/2020.11.04.355842 (2020). 